\title{
手性胺修饰的羟基磷灰石负载 $\mathrm{RuCl}_{2}(\mathrm{TPP})_{3}$ 催化不对称氢化苯乙酮
}

\author{
张定林 ${ }^{1,2}$ 杨朝芬 $^{1} \quad$ 孙亚萍 $^{1} \quad$ 付海燕 $^{1}$ \\ 李瑞祥 ${ }^{1}$ 陈 华 ${ }^{1, *}$ 李贤均 ${ }^{1}$ \\ ( ${ }^{1}$ 四川大学化学学院, 有机金属络合催化研究所, 绿色化学与技术教育部重点实验室, 成都 610064; \\ 2 第三军医大学药学院化学教研室, 重庆 400038)
}

\begin{abstract}
摘要：采用共沉淀法制备了手性胺(L-脯氨酸、D-脯氨酸、( $1 R, 2 R)-1,2$-二苯基乙二胺二磺酸钠 $((1 R, 2 R)-\mathrm{DPENDS})$ 、 $(1 S, 2 S)-1,2$ 二 二苯基乙二胺二磺酸钠((1S,2S)-DPENDS))修饰的羟基磷灰石(HAP). 并采用傅里叶变换红外(FT-IR) 光谱, 扫描电子显微镜(SEM), $X$ 射线衍射(XRD)和比表面积测定(BET)等仪器分析手段对其进行表征. 以手性 胺修饰的羟基磷灰石做载体负载 $\mathrm{RuCl}_{2}(\mathrm{TPP})_{3}$ 催化苯乙酮不对称加氢反应, 详细考察温度、压力、碱的浓度、手性 胺负载量等条件对催化反应的影响. 在氢气压力为 $5.0 \mathrm{MPa} 、 30{ }^{\circ} \mathrm{C}$ 条件下反应 $4 \mathrm{~h}$, 苯乙酮的不对称加氢反应, 可 获得 $99.9 \%$ 转化率和 $77.8 \%$ 对映选择性, 其结果优于对应的均相催化反应. 实验结果证明, 催化反应在载体表面 完成, 催化剂通过简单离心分离可循环使用.
\end{abstract}

关键词：手性胺；羟基磷灰石； $\mathrm{RuCl}_{2}(\mathrm{TPP})_{3}$ ；苯乙酮；多相不对称加氢 中图分类号： O643; O641; O433

\section{Asymmetric Hydrogenation of Acetophenone Catalyzed by $\operatorname{RuCl}_{2}(\mathrm{TPP})_{3}$ Supported on Hydroxyapatite Modified with Chiral Amine}

\author{
ZHANG Ding-Lin ${ }^{1,2} \quad$ YANG Chao-Fen $^{1} \quad$ SUN Ya-Ping ${ }^{1} \quad$ FU Hai-Yan ${ }^{1}$ \\ LI Rui-Xiang ${ }^{1} \quad$ CHEN Hua ${ }^{1, *} \quad$ LI Xian-Jun ${ }^{1}$
}

('Key Laboratory of Green Chemistry and Technology, Ministry of Education, Institute of Homogeneous Catalysis, College of Chemistry, Sichuan University, Chengdu 610064, P. R. China; ${ }^{2}$ Department of Chemistry, School of Pharmacy, The Third Military Medical University, Chongqing 400038, P. R. China)

\begin{abstract}
Chiral amine (L-proline, D-proline, (1R,2R)-1,2-diphenyl-1,2-ethylene diamine sulfonate disodium (( $1 R$, $2 R)$-DPENDS) and (1S,2S)-1,2-diphenyl-1,2-ethylene diamine sulfonate disodium ((1S,2S)-DPENDS)) modified hydroxyapatite (HAP) were prepared and characterized by Fourier transform infrared (FT-IR) spectrometry, scanning electron microscopy (SEM), X-ray diffraction (XRD), and Brunauer-Emmett-Teller (BET) surface area analysis. $\mathrm{RuCl}_{2}$ $(\mathrm{TPP})_{3}$ was supported on $(1 R, 2 R)$-DPENDS modified HAP for the asymmetric hydrogenation of acetophenone. A detailed investigation about the effects of temperature, amount of alkali, $\mathrm{H}_{2}$ pressure, and concentration of DPENDS modified HAP on the activity and enantioselectivity of acetophenone hydrogenation was undertaken. Under the optimum reaction conditions of $30{ }^{\circ} \mathrm{C}, p\left(\mathrm{H}_{2}\right)=5.0 \mathrm{MPa}$, reaction time of $4 \mathrm{~h}$, the conversion and enantioselectivity reached $99.9 \%$ and $77.8 \%$ enantiomeric excess (ee), respectively. The results indicated that the asymmetric hydrogenation was accomplished on the surface of the chiral amine modified HAP. The immobilized catalyst was separated from the products by simple centrifugation and reused without adding any other reagents.
\end{abstract}

Key Words: Chiral amine; Hydroxyapatite; $\mathrm{RuCl}_{2}(\mathrm{TPP})_{3} ; \quad$ Acetophenone; Heterogeneous asymmetric hydrogenation

Received: April 22, 2010; Revised: June 7, 2010; Published on Web: September 3, 2010.

*Corresponding author. Email: scuhchen@163.com; Tel/Fax: +86-28-85412904

C Editorial office of Acta Physico-Chimica Sinica 
前手性酮的不对称加氢是获得手性仲醇的重要 方法之一. 在对简单芳香酮的不对称加氢反应中, 均 相催化剂可以获得高转化率和高选择性 ${ }^{[1-2]}$, 但是由 于在均相催化反应体系中, 催化剂与底物分离困难 等限制了其实际应用. 多相不对称加氢反应为解决 上述问题提供了一个可行的方法. 对简单芳香酮的 多相不对称加氢反应的研究目前主要集中在手性修 饰剂修饰的负载金属催化剂和将均相催化剂多相化 这两个方面. 在手性修饰剂修饰的负载金属催化剂 对简单芳香酮的不对称加氢反应中, Baiker 等 ${ }^{[3]}$ 成功 利用金鸡纳碱修饰 $\mathrm{Pt} / \mathrm{Al}_{2} \mathrm{O}_{3}$ 对活化的芳香酮进行不 对称加氢反应. Cheng 等 ${ }^{[4]}$ 以 $\mathrm{Ru} / \mathrm{Al}_{2} \mathrm{O}_{3}$ 为催化剂, 原 位加人三苯基膦(TPP)和 $(1 R, 2 R)-1,2$-二苯基乙二胺 $[(1 R, 2 R)-\mathrm{DPEN}]$ 催化苯乙酮不对称加氢反应, 获得 了 $60.5 \%$ 的对映选择性(ee)值. 本课题组在 $\mathrm{Ru} / \mathrm{Al}_{2} \mathrm{O}_{3}$ 和 $\mathrm{Ir} / \mathrm{Al}_{2} \mathrm{O}_{3}$ 的制备过程中加人 TPP 作为稳定剂, 以 $(1 S, 2 S)-1,2$-二苯基乙二胺 $[(1 S, 2 S)$-DPEN $]$ 和 $(8 S, 9 S)$ 9-氨基(9-脱氧)辛可尼定为手性修饰剂时, 催化苯乙 酮的不对称加氢反应, 分别获得了 $77.7 \%$ 和 $95.0 \%$ 的 ee 值 ${ }^{[5-9]}$; 而辛可尼定稳定的 $\mathrm{Ir} / \mathrm{SiO}_{2}$ 催化剂, 以( $1 S$, $2 S$ )-DPEN 为手性修饰剂, 可获得 $79.8 \%$ 的 ee 值 ${ }^{[7]}$; 采用羟基磷灰石负载的铱催化剂, 以( $1 S, 2 S)$-DPEN 为手性修饰剂, 可获得 $63.7 \%$ 的 ee 值 ${ }^{[8]}$. 然而在上述 催化反应体系中, 催化剂循环使用时, 手性二胺的流 失不可避免. 在对简单芳香酮的多相不对称加氢反 应的另一重要方法就是将均相手性催化剂通过共价 键链接到聚合物 ${ }^{[0-13]}$ 或无机载体上 ${ }^{[14-18]}$, 以达到均相 催化剂多相化的目的. 通过该方法制备的多相催化 剂具备均相催化剂的高活性、高选择性的优点, 同时 也具备多相催化剂的分离方法简单、可循环使用的 优点, 不足之处就是催化剂的制备方法比较复杂, 需 要多步合成. 因而发展一种简单的催化剂制备方法, 以实现均相催化剂多相化的目的, 有其重要意义.

差基磷灰石(HAP)表面具有丰富的羟基,而且 具有强吸附性, 能被带极性基团的有机化合物所修 饰. 已有文献报道利用烷基磷酸 ${ }^{[19-22]}$ 、十二烷基醇 ${ }^{[23]}$ 、

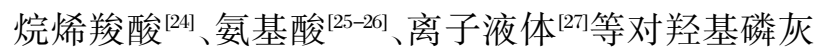
石表面进行修饰. Baiker 等 ${ }^{[2]}$ 利用脯氨酸、苯甲酸等 对羟基磷灰石表面进行修饰, 然后负载金属钓催化 醇的氧化反应, 发现催化活性是未修饰前的三倍, 他 们认为钉负载到有机物修饰的羟基磷灰石上不是通 过离子交换, 而是通过与羟基磷灰石上的有机物配 位来实现的. 而采用简单的吸附方法利用带极性基

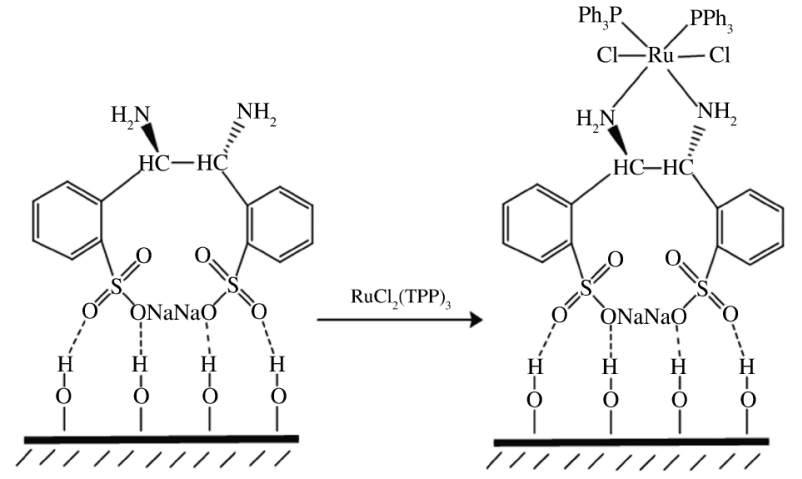

图 1 手性胺修饰的羟基磷灰石(HAP)负载均相催化剂 $\mathrm{RuCl}_{2}(\mathbf{T P P})_{3}$

Fig.1 Immobilized the homogenous catalyst $\mathrm{RuCl}_{2}(\mathrm{TPP})_{3}$ on chiral amine modified hydroxyapatite (HAP)

团的手性胺修饰无机载体, 然后再负载有机金属络 合物对简单芳香酮进行多相不对称加氢反应的方法 未见报道. 本文通过共沉淀的方法制备了手性胺 $(\mathrm{L}-$ 脯氨酸、D-脯氨酸、 $(1 R, 2 R)-1,2-$ 二苯基乙二胺二磺 酸钠 $[(1 R, 2 R)$-DPENDS $] 、(1 S, 2 S)-1,2$-二苯基乙二胺 二磺酸钠) $[(1 S, 2 S)$-DPENDS $]$ 修饰的羟基磷灰石, 再 利用手性胺修饰的羟基磷灰石原位负载 $\mathrm{RuCl}_{2}(\mathrm{TPP})_{3}$ (图 1), 催化苯乙酮的不对称加氢反应, 以达到通过 简单催化剂制备方法而实现均相催化剂多相化的目 的.

\section{1 实验部分}

\section{1 试剂与仪器}

$\mathrm{RuCl}_{3} \cdot 3 \mathrm{H}_{2} \mathrm{O}$ (昆明贵金属研究所, 分析纯), $\mathrm{Ca}\left(\mathrm{NO}_{3}\right)_{2} \cdot 4 \mathrm{H}_{2} \mathrm{O}$ 和 $\left(\mathrm{NH}_{4}\right)_{2} \mathrm{HPO}_{4}$ (成都科龙试剂有限公 司, 分析纯), L-脯氨酸或 D-脯氨酸(>99.5\%, 爱斯特 试剂有限公司), 苯乙酮( $\geqslant 98 \%$, Acros 公司, 比利时), 三苯基膦和其它试剂为分析纯. 三苯基膦使用前用 乙醇重结晶. 高纯氢(99.99\%); (1R,2R)-DPENDS(示意 图 1)或(1S,2S)-DPENDS 通过磺化( $(1 R, 2 R)$-DPEN 或 $(1 S, 2 S)$-DPEN (成都丽凯手性试剂公司, $>99 \%$ ) ${ }^{[29]}$ 制 得.<smiles>N[C@H]1c2ccccc2ONOc2ccccc2[C@@H](N)[C@H]1N</smiles>

示意图 $1(1 R, 2 R)-1,2$-二苯基乙二胺二磺酸钠结构式 Scheme 1 Structure of $(1 R, 2 R)$-DPENDS 
催化剂的 XRD 测试在日本 Rigaku 理学公司 RINT 2500X 型 X 射线衍射仪上进行, $\mathrm{Cu} K_{\alpha}$ 射线. BET 表面积通过低温氮吸附方法在 NOVAl200e 型 物理吸附仪(美国 Quantachrome 公司)上进行. 载体 的形貌用日本电子株式会社 JSM-5900LV 扫描电镜 观察. 红外光谱图用 thermo nicolet IR 红外光谱仪 (美国热电尼高力)分析. 钉含量分析用等离子发射 光谱(ICP)(美国 Thermo Elementa 公司)测定. 底物 转化率和产物的对映选择性用 GC-960 气相色谱仪 (上海海欣色谱仪器有限公司) 分析, 手性毛细管色 谱柱为 $\beta-\mathrm{CD}^{\mathrm{TM}}(30 \mathrm{~m} \times 0.25 \mathrm{~mm} \times 0.15 \mu \mathrm{m}$, 美国Supelco 公司), 色谱柱温度为 $120{ }^{\circ} \mathrm{C}$, 氢火焰离子化检测器.

\section{2 手性胺修饰的羟基磷灰石及 $\mathrm{RuCl}_{2}(\mathrm{TPP})_{3}$ 的 制备}

$\mathrm{RuCl}_{2}(\mathrm{TPP})_{3}$ 按文献[30]方法合成: 将 $0.2 \mathrm{~g}(0.76$ $\mathrm{mmol}) \mathrm{RuCl}_{3} \cdot 3 \mathrm{H}_{2} \mathrm{O}$ 溶解到去氧甲醇中 $(50 \mathrm{~mL})$, 在氩 气保护下回流 $5 \mathrm{~min}$ 得红棕色溶液. 冷却后, 加人 $1.2 \mathrm{~g}(4.58 \mathrm{mmol})$ 三苯基膦, 在氩气保护下回流 $3 \mathrm{~h}$, 得红棕色固体. 冷却后, 在氩气保护下过滤, 用脱氧 乙醚洗固体三次, 真空干燥, 得 $\mathrm{RuCl}_{2}(\mathrm{TPP})_{3}$ 配合物.

手性胺修饰的羟基磷灰石按参考文献方法制 备 ${ }^{[28,31]}$. 按 $\mathrm{Ca}\left(\mathrm{NO}_{3}\right)_{2}:\left(\mathrm{NH}_{4}\right)_{2} \mathrm{HPO}_{4}:(1 R, 2 R)-\mathrm{DPENDS}$ 摩 尔比为 1:0.6:0.08 投料. 硝酸钻和 $(1 R, 2 R)$-DPENDS 溶解于去离子水中, 用氨水调 $\mathrm{pH}=10$ 左右; 将磷酸 氢二铵溶解于去离子水中, 用氨水调 $\mathrm{pH}=10$ 左右, 在快速摚拌下将磷酸氢二胺溶液向含有 $(1 R, 2 R)$ DPENDS 的硝酸钙溶液中滴加, 滴加完后微沸 10 $\mathrm{min}$, 静置老化 $4 \mathrm{~h}$, 过滤, 去离子水洗至近中性, 100 ${ }^{\circ} \mathrm{C}$ 真空干燥 $17 \mathrm{~h}$, 得手性胺修饰的差基磷灰石, 缩写 为 $8 \%(1 R, 2 R)$-DPENDS/HAP. $4 \%(1 R, 2 R)$-DPENDS/ HAP 或 $4 \%(1 S, 2 S)$-DPENDS/HAP 用同样的方法制 备. 脯氨酸修饰的羟基磷灰石按 $\mathrm{Ca}\left(\mathrm{NO}_{3}\right)_{2}:\left(\mathrm{NH}_{4}\right)_{2} \mathrm{HPO}_{4}$ : 脯氨酸摩尔比为 1:0.6:0.3 投料, 并缩写为 L-proline/ HAP 或 D-proline/HAP.

\section{3 芳香酮不对称加氢反应}

不对称加氢反应在带磁力搅拌的高压釜中进 行, 其反应式见示意图 2. 将 $\mathrm{RuCl}_{2}(\mathrm{TPP})_{3}$ 、手性胺修 饰的 HAP、 $\mathrm{KOH}$ 、异丙醇和底物加人到高压釜中, 用 高纯氢置换 4 次, 氢气加到预定压力在预定温度反 应 $4 \mathrm{~h}$. 反应完成后冷却, 解除压力, 通过离心分离分 出催化剂, 反应液用气相色谱分析底物转化率和产 物对映选择性(ee). 产物的 ee 值按下式计算:

$$
\text { ee }=[C(R)-C(S)] /[C(R)+C(S)] \times 100 \%
$$

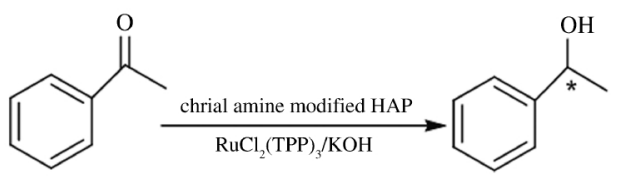

示意图 2 苯乙酮的不对称加氢反应反应式

Scheme 2 Asymmetric hydrogenation reaction of acetophenone

其中 $C(R)$ 代表 $R$ 构型产物的质量分数, $C(S)$ 代表 $S$ 构型产物的质量分数.

\section{2 结果与讨论}

\section{1 催化剂的表征}

\subsection{1 手性胺修饰的羟基磷灰石的红外光谱分析}

羟基磷灰石和手性胺修饰的羟基磷灰石的红外 光谱图如图 2 所示. 在羟基磷灰石和手性胺修饰的 羟基磷灰石中, 3570 和 $630 \mathrm{~cm}^{-1}$ 为羟基磷灰石晶格 中的羟基振动 ${ }^{[28]}$; 3430 和 $1629 \mathrm{~cm}^{-1}$ 为羟基磷灰石 表面吸附的水的振动(图 2(A)). $\mathrm{PO}_{4}^{3-}$ 的特征吸收峰 出现在 1094、1039、963、603 和 $568 \mathrm{~cm}^{-1}$, 这与文献 报道 ${ }^{[22] 一}$ 致. 对比研究手性胺修饰的差基磷灰石和 未修饰的羟基磷灰石红外谱图,一系列特征吸收峰 出现在手性胺修饰的羟基磷灰石中. 在 $(1 R, 2 R)$ DPENDS 修饰的差基磷灰石中, 1384 和 $1414 \mathrm{~cm}^{-1}$ 吸收峰为苯环的伸缩振动(图 2(B, C), $875 \mathrm{~cm}^{-1}$ 吸收 峰为 $\mathrm{C}-\mathrm{H}$ 伸缩振动. 在脯氨酸修饰的差基磷灰石 中, 1462 和 $1411 \mathrm{~cm}^{-1}$ 为脯氨酸中羰基的伸缩振动 和弯曲振动(图 2(D)). 红外谱图分析结果表明, 手性 胺被成功地负载到载体表面, 并对羟基磷灰石表面

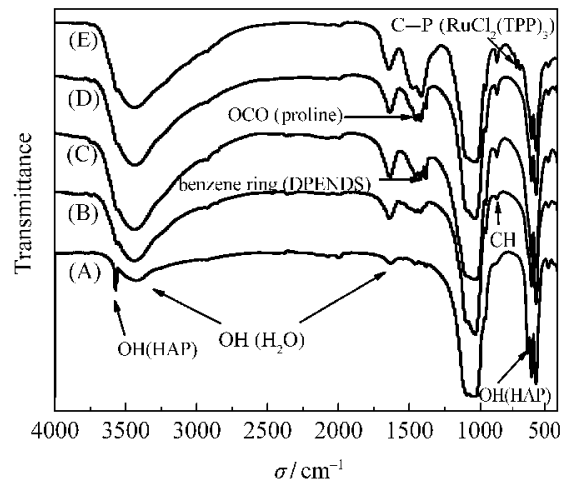

图 2 HAP (A), 4\% (1R,2R)-DPENDS/HAP (B), 8\% $(1 R, 2 R)$-DPENDS/HAP $(C)$, L-脯氨酸/HAP (D) 和 $8 \%$

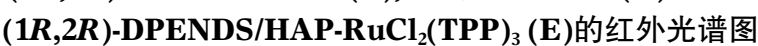

Fig.2 IR spectra of HAP (A), 4\% (1R,2R)-DPENDS/

HAP (B), $8 \%(1 R, 2 R)$-DPENDS/HAP (C), L-proline/HAP (D) and $8 \%(1 R, 2 R)$-DPENDS/HAP- $\mathrm{RuCl}_{2}(\mathrm{TPP})_{3}(\mathrm{E})$ 
进行修饰. 然而, 在手性胺修饰的羟基磷灰石中, 差 基磷灰石晶格中的羟基振动吸收峰(3570 和 630 $\mathrm{cm}^{-1}$ )变弱(图 2(B-E), 表明脯氨酸的羧基和DPENDS 的磺酸根与羟基磷灰石表面的羟基发生了相互作 用, 可能形成了氢键. 在手性胺修饰的羟基磷灰石负 载了 $\mathrm{RuCl}_{2}(\mathrm{TPP})_{3}$ 后, 苯环的吸收峰加强, 且在 721 $\mathrm{cm}^{-1}$ 处出现一个新的吸收峰, 归属为 $\mathrm{RuCl}_{2}(\mathrm{TPP})_{3}$ 中的 $\mathrm{C}-\mathrm{P}$ 振动(图 2(E)). 结果表明 $\mathrm{RuCl}_{2}(\mathrm{TPP})_{3}$ 成 功负载到手性胺修饰的着基磷灰石上.

\subsection{2 手性胺修饰的羟基磷灰石的 SEM 和 BET 分析}

差基磷灰石和手性胺修饰的羟基磷灰石的 SEM 分析结果见图 3. 未加手性胺修饰的羟基磷灰 石表面多孔, 且颗粒的平均尺寸比较均匀(图 3(A)). 加人手性胺后, 明显改变羟基磷灰石的表面形态, 手 性胺修饰的差基磷灰石表面变得紧密, 而且随着手 性胺浓度的增加, 表面变得更加紧密(图 3(B-D)). 从 图 3 还可知, 手性胺修饰的差基磷灰石颗粒的平均 尺寸有所减小, 而且随着手性胺浓度增加, 颗粒减小 明显. 该结果与文献报道的其它有机物修饰的差基 磷灰石结果类似 ${ }^{[28]}$. 表明手性胺对载体表面产生明 显影响, 手性胺成功负载到了载体表面, 与红外光谱 分析一致.

表 1 列出了羟基磷灰石和手性胺修饰的羟基磷 灰石的比表面积及孔径分析结果. 由表 1 可知, 随着 手性胺的引人, 差基磷灰石的孔径变小, 且成规律性
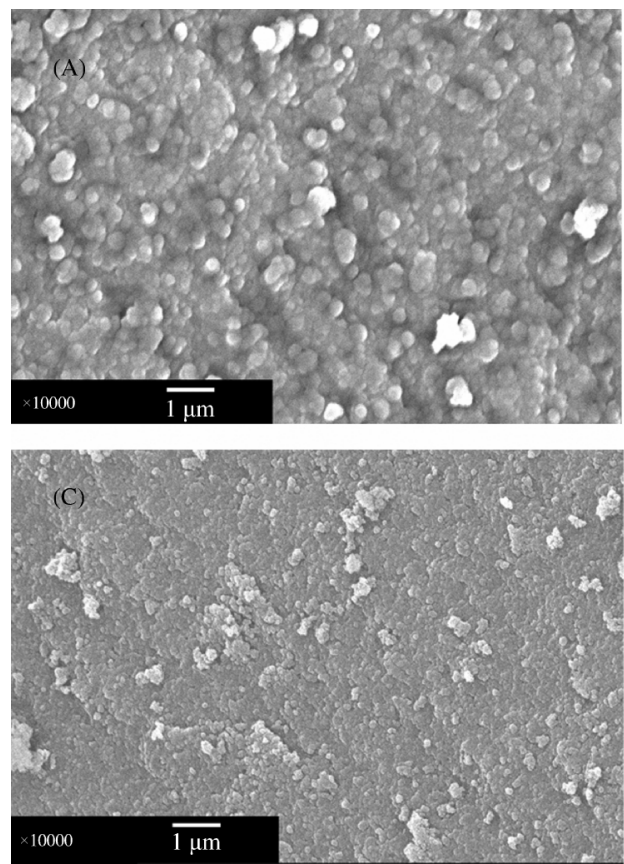

表 1 手性胺修饰的羟基磷灰石的 BET 表征 Table 1 Characterization of HAP modified with a chiral amine by BET measurement

\begin{tabular}{lcc}
\hline & $d_{\text {por }} / \mathrm{nm}$ & $S_{\mathrm{BEI}} /\left(\mathrm{m}^{2} \cdot \mathrm{g}^{-1}\right)$ \\
\hline HAP & 23 & 62 \\
$4 \%(1 R, 2 R)$-DPENDS/HAP & 15 & 125 \\
$8 \%(1 R, 2 R)$-DPENDS/HAP & 14 & 145 \\
proline/HAP & 12 & 168 \\
\hline
\end{tabular}

变化, 即手性胺浓度增加, 载体的孔径减小. 可能是 手性胺的引人导致载体的平均粒度减小, 使得载体 排列更紧密, 载体的孔径减小. 而随着手性胺的引 人, 载体的比表面积明显增加, 从 $62 \mathrm{~m}^{2} \cdot \mathrm{g}^{-1}$ 增加到 $125 \mathrm{~m}^{2} \cdot \mathrm{g}^{-1}$, 特别是引人脯氨酸后, 其比较面积达到 $168 \mathrm{~m}^{2} \cdot \mathrm{g}^{-1}$, 这与 $\mathrm{SEM}$ 分析结果一致.

2.1.3 手性胺修饰的羟基磷灰石的 XRD 分析

对手性胺修饰的羟基磷灰石和未修饰的羟基磷 灰石进行了 XRD 分析, 结果如图 4 所示. 空白羟基 磷灰石的 XRD 图峰型比较尖锐, 说明制备的差基 磷灰石结晶比较好. 在加人手性胺后, 羟基磷灰石的 特征峰没有明显改变, 也未出现新的特征峰, 但是手 性胺修饰的羟基磷灰石的 XRD 峰型明显变宽, 说 明手性胺的引人使得载体的晶形变差, 其原因见 SEM 及 BET 分析结果.

\section{2 苯乙酮的不对称催化加氢反应}

\subsection{1 氢气压力对反应的影响}

以 $8 \%(1 R, 2 R)$-DPENDS/HAP 负载 $\mathrm{RuCl}_{2}(\mathrm{TPP})_{3}$
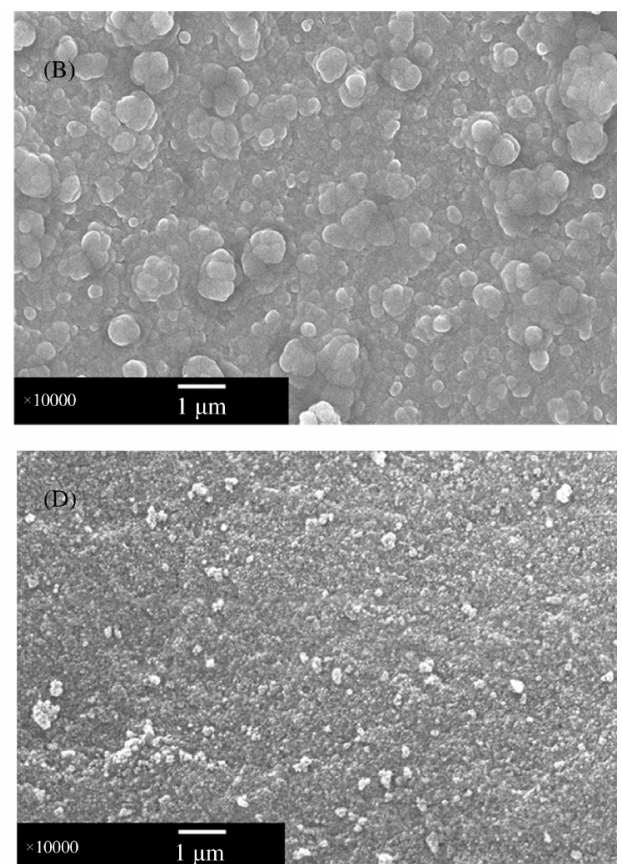

图 3 HAP $(\mathrm{A}), 4 \%(1 R, 2 R)$-DPENDS/HAP $(\mathrm{B}), 8 \%(1 R, 2 R)-D P E N D S / H A P(C)$ 和 L-脯氨酸/HAP (D) 的 SEM 图

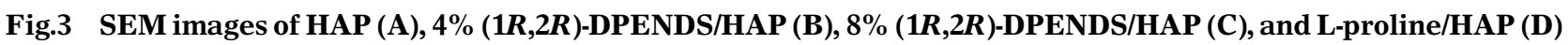




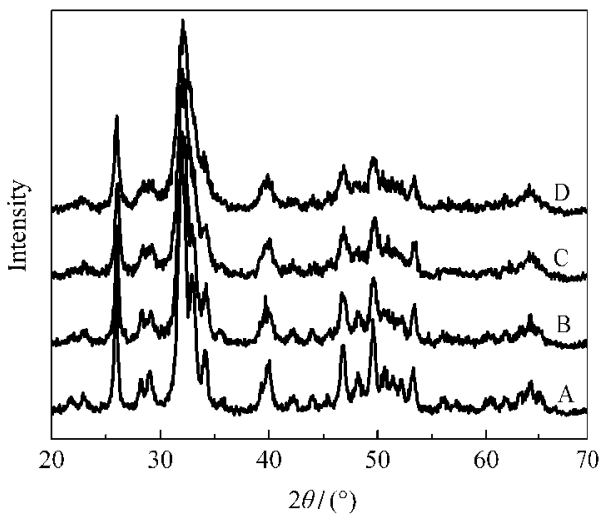

图 4 HAP (A), 4\% (1R,2R)-DPENDS/HAP (B), 8\% $(1 R, 2 R)-D P E N D S / H A P(C)$ 和 L-脯氨酸/HAP $(D)$ 的 XRD 图

Fig.4 XRD patterns of HAP (A), 4\% (1R,2R)DPENDS/HAP (B), $8 \%(1 R, 2 R)$-DPENDS/HAP (C), and L-proline/HAP (D)

为催化剂时氢气压力对苯乙酮加氢反应的影响如图 5 所示. 由图 5 可知, 底物的转化率随压力的升高而 增加, 产物的 ee 值随压力的升高变化不是很明显. 在 $2 \mathrm{MPa}$ 时, 底物苯乙酮的转化率只有 $61.6 \%$, 产物 的 ee 值为 $68.2 \%$. 而当压力增加到 $3 \mathrm{MPa}$ 时候, 反 应活性明显增加, 底物转化率从 $61.6 \%$ 上升至 $99.0 \%$, 产物的 ee 值为 $70.5 \%$. 继续增加氢气压力到 $5 \mathrm{MPa}$ 时, 产物的 ee 值增加到 $73.1 \%$, 此 ee 值相对 比较高, 在该不对称加氢体系中是最适合的反应压 力.

\subsection{2 温度对反应的影响}

以 $8 \%(1 R, 2 R)$-DPENDS/HAP 负载 $\mathrm{RuCl}_{2}(\mathrm{TPP})_{3}$ 为催化剂时, 反应温度对苯乙酮加氢的影响见图 6.

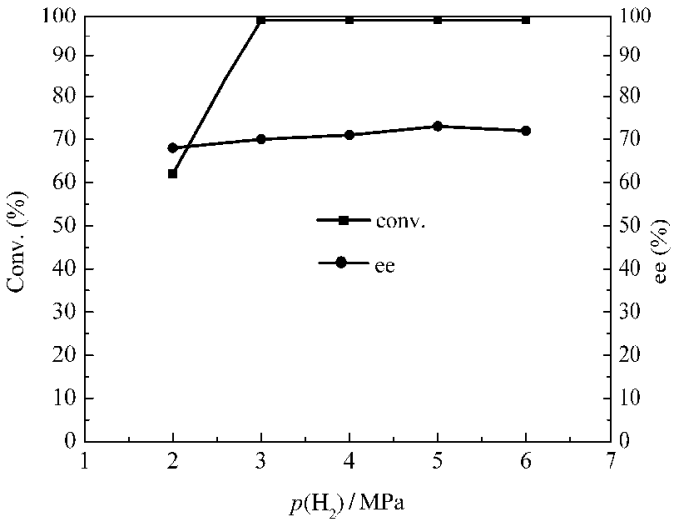

图 5 压力对苯乙酮不对称加氢反应的影响

Fig.5 Effect of the pressure on asymmetric hydrogenation of acetophenone

reaction conditions: $20 \mathrm{mg} 8 \%(1 R, 2 R)$-DPENDS modified HAP,

$4 \mathrm{mg} \mathrm{RuCl}{ }_{2}(\mathrm{TPP})_{3}, c_{\mathrm{KoH}}=9 \times 10^{-2} \mathrm{~mol} \cdot \mathrm{L}^{-1}, n$ (acetophenone $)=0.43 \mathrm{mmol}$, $V(2$-propanol $)=2.0 \mathrm{~mL}, T=313 \mathrm{~K}, t=4 \mathrm{~h}$

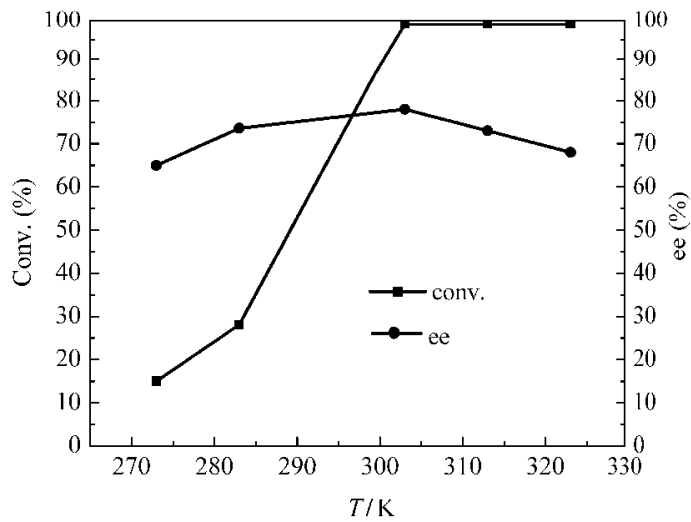

图 6 温度对苯乙酮不对称加氢反应的影响

Fig.6 Effect of the temperature on asymmetric hydrogenation of acetophenone

reaction conditions: $20 \mathrm{mg} 8 \%(1 R, 2 R)$-DPENDS modified HAP, $4 \mathrm{mg} \mathrm{RuCl}{ }_{2}(\mathrm{TPP})_{3}, c_{\mathrm{KoH}}=9 \times 10^{-2} \mathrm{~mol} \cdot \mathrm{L}^{-1}, n$ (acetophenone $)=0.43 \mathrm{mmol}$, $V(2$-propanol $)=2.0 \mathrm{~mL}, p\left(\mathrm{H}_{2}\right)=5 \mathrm{MPa}, t=4 \mathrm{~h}$

对于该催化剂体系, 并不是温度越低获得的 ee 值越高, 在 $273 \mathrm{~K}$ 时转化率仅为 $15.0 \%$, 而产物的 ee 值也只有 $64.5 \%$, 其原因可能是在温度低时, 催化剂 反应活性低, 故底物转化率和产物的 ee 值都比较 低, 同样的现象在文献中也有报道 ${ }^{[]}$. 当温度升到 303 $\mathrm{K}$ 后, 底物的转化率高达 $99.3 \%$, 而产物的 ee 也上 升到 $75.0 \%$. 说明温度的升高有利于手性胺与中心 金属的配位, 导致催化剂活性提高, 产物的 ee 值增 加. 当温度超过 $303 \mathrm{~K}$ 后, 继续升高温度, 产物的 ee 值反而下降, 其原因是随着温度的进一步升高, 生成 两个对映异构体的活化能差变小, 导致产物的对映 选择性下降. 由图 6 可知, $303 \mathrm{~K}$ 是该催化体系的最 佳反应温度.

\subsection{3 碱浓度对反应的影响}

图 7 给出了 $\mathrm{KOH}$ 用量对 $8 \%(1 R, 2 R)$-DPENDS/ $\mathrm{HAP}$ 负载 $\mathrm{RuCl}_{2}(\mathrm{TPP})_{3}$ 催化苯乙酮加氢反应的影响. 当没有 $\mathrm{KOH}$ 加人时, 催化剂没有活性. 而 $\mathrm{KOH}$ 的 浓度为 $4.5 \times 10^{-2} \mathrm{~mol} \cdot \mathrm{L}^{-1}$ 时, 底物的转化率可以达到 $81.6 \%$, 产物的对映选择性可以达到 $68.9 \%$, 这说明 碱的加人明显提高了催化剂的活性和产物的对映选 择性. 当 $\mathrm{KOH}$ 的浓度达到 $9 \times 10^{-2} \mathrm{~mol} \cdot \mathrm{L}^{-1}$ 时, 可以 获得 $99.9 \%$ 的转化率和 $77.8 \%$ 的 ee 值, 其产物的对 映选择性达到最大值. 随着 $\mathrm{KOH}$ 浓度的进一步增 加, 产物的对映选择性有所下降. 故在该反应体系 中, 当 $\mathrm{KOH}$ 的浓度为 $9 \times 10^{-2} \mathrm{~mol} \cdot \mathrm{L}^{-1}$ 时, 可以获得 较高的催化剂活性和较好的产物对映选择性.

\subsection{4 手性胺不同负载量对反应的影响}

对比研究了空白羟基磷灰石、不同手性修饰剂负 


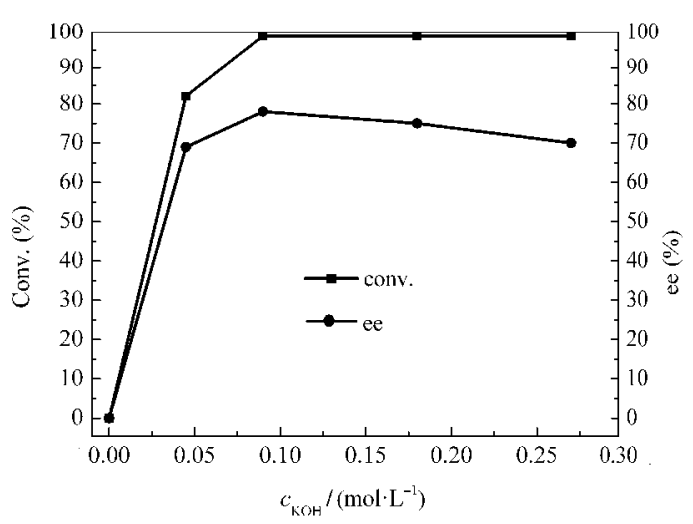

图 $7 \mathrm{KOH}$ 的浓度对苯乙酮的不对称加氢的影响

Fig.7 Effect of the concentration of $\mathrm{KOH}$ on asymmetric hydrogenation of acetophenone reaction conditions: $20 \mathrm{mg} 8 \%$ ( $1 R, 2 R$ )-DPENDS modified HAP, $4 \mathrm{mg}$ $\mathrm{RuCl}_{2}(\mathrm{TPP})_{3}, n$ (acetophenone $)=0.43 \mathrm{mmol}, V(2$-propanol $)=2.0 \mathrm{~mL}$, $p\left(\mathrm{H}_{2}\right)=5 \mathrm{MPa}, T=303 \mathrm{~K}, t=4 \mathrm{~h}$

载量的羟基磷灰石负载 $\mathrm{RuCl}_{2}(\mathrm{TPP})_{3}$ 的催化性能, 结 果见表 2 . 当使用空白的羟基磷灰石负载 $\mathrm{RuCl}_{2}(\mathrm{TPP})_{3}$ 催化苯乙酮加氢反应时, 底物的转化率为 $25.0 \%$, 产 物为外消旋体. 当使用 $4 \%(1 R, 2 R)$-DPENDS/HAP 负 载 $\mathrm{RuCl}_{2}(\mathrm{TPP})_{3}$ 为催化剂时, 可以获得 $53.0 \%$ 的转 化率和 $51.0 \%$ 的 ee 值, 这说明手性胺成功负载到 了羟基磷灰石上, 实验结果与前面对载体的仪器 表征结果一致. 当使用 $8 \%(1 R, 2 R)$-DPENDS/HAP 负 载 $\mathrm{RuCl}_{2}(\mathrm{TPP})_{3}$ 为催化剂时, 底物的转化率高达 99.9\%, 产物的对映选择性达到 $77.8 \%$, 其催化活性 和选择性明显高于 $4 \%(1 R, 2 R)$-DPENDS/HAP 负载 $\mathrm{RuCl}_{2}(\mathrm{TPP})_{3}$ 的催化反应结果, 略高于使用三苯基膦 的均相催化剂对苯乙酮的不对称加氢的结果 (75.0\% 的 ee 值). 这说明在该催化反应体系中, 底 物的转化率和产物的对映选择性都依赖于手性胺在 羟基磷灰石表面的浓度. 在该催化反应体系中, 由于 $(1 R, 2 R)$-DPENDS 在异丙醇中不溶解, 说明对苯乙酮

表 2 DPENDS 在羟基磷灰石中负载量对不对称加氢的影响

Table 2 Influence of DPENDS concentration supported by HAP on asymmetric hydrogenation reaction

\begin{tabular}{|c|c|c|c|}
\hline Catalyst & Conv. $(\%)$ & ee $(\%)$ & Configuration \\
\hline HAP- $\mathrm{RuCl}_{2}(\mathrm{TPP})_{3}$ & 25.0 & - & - \\
\hline $4 \%(1 R, 2 R)$-DPENDS/HAP- $\mathrm{RuCl}_{2}(\mathrm{TPP})_{3}$ & 53.0 & 51.0 & $S$ \\
\hline $4 \%(1 S, 2 S)$-DPENDS/HAP-RuCl ${ }_{2}(\mathrm{TPP})_{3}$ & 53.8 & 52.3 & $R$ \\
\hline $8 \%(1 R, 2 R)$-DPENDS/HAP-RuCl${ }_{2}(\mathrm{TPP})_{3}$ & 99.9 & 77.8 & $S$ \\
\hline $\begin{array}{l}\text { homogenous catalyst } \\
(1 R, 2 R)-\mathrm{DPEN}-\mathrm{RuCl}_{2}(\mathrm{TPP})_{3}\end{array}$ & 99.9 & 75.0 & $S$ \\
\hline
\end{tabular}

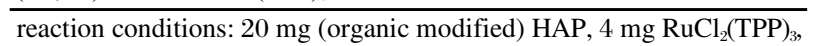
$c_{\mathrm{KOH}}=9 \times 10^{-2} \mathrm{~mol} \cdot \mathrm{L}^{-1}, n($ acetophenone $)=0.43 \mathrm{mmol}, V(2$-propanol $)=2.0$ $\mathrm{mL}, p\left(\mathrm{H}_{2}\right)=5 \mathrm{MPa}, T=303 \mathrm{~K}, t=4 \mathrm{~h}$
的不对称加氢反应在载体表面完成, 而不是在溶液 中进行的.

\section{$2.2 .54 \%(1 R, 2 R)-\mathrm{DPENDS} / \mathrm{HAP}$ 与中心金属不同摩尔比对 反应的影响}

为了进一步研究手性胺修饰的羟基磷灰石对催 化剂性能影响, 采用 $4 \%(1 R, 2 R)$-DPENDS/HAP 为载 体负载 $\mathrm{RuCl}_{2}(\mathrm{TPP})_{3}$, 通过改变手性胺修饰的羟基磷 灰石的用量来控制手性胺和中心金属的比例, 其催 化苯乙酮不对称加氢反应的结果见表 3 . 当 $5 \mathrm{mg}$ 的 $4 \%(1 R, 2 R)$-DPENDS/HAP 用于负载 $\mathrm{RuCl}_{2}(\mathrm{TPP})_{3}$ 时, $(1 R, 2 R)$-DPENDS 与 $\mathrm{Ru}$ 的理论摩尔比为 $0.5: 1$, 苯乙 酮的转化率可达到 $30.6 \%$, 而产物的 ee 值只有 $27.1 \%$. 增加载体用量使手性胺与 $\mathrm{Ru}$ 的摩尔比为 1 : 1 时, 苯乙酮的转化率增加到 $34.1 \%$, 产物 ee 值上升 为 $37.0 \%$; 而当手性胺与 $\mathrm{Ru}$ 的摩尔比达到 $3: 1$ 时, 苯乙酮转化率达到 $81.5 \%$, ee 值达到 $58.3 \%$. 手性胺 用量进一步增加到与 $\mathrm{Ru}$ 的摩尔比达到 4:1 时, 底物 的转化率和产物的对映选择性反而下降, 分别为 $78.2 \%$ 和 $56.1 \%$. 而采用 $8 \%(1 R, 2 R)$-DPENDS/HAP 做 载体, 当手性胺与 $\mathrm{Ru}$ 的比例达到 4:1 时, 底物的转 化率达到 99.9\%, 产物的对映选择性达到 $77.8 \%$, 明 显高于 $4 \%(1 R, 2 R)$-DPENDS/HAP 做载体时的活性 和选择性. 这进一步说明在该催化反应体系中, 手性 胺的负载量对催化剂的性能有重要影响, 进一步证

\section{表 $34 \%(1 S, 2 S)$-DPENDS/HAP 的用量对苯乙酮不对称 加氢的影响}

Table 3 Influence of the dosage of $4 \%(1 S, 2 S)$ DPENDS/HAP on asymmetric hydrogenation reaction

\begin{tabular}{ccccc}
\hline $\begin{array}{c}m(4 \%(1 \mathrm{~S}, 2 \mathrm{~S})- \\
\text { DPENDS/HAP)/mg }\end{array}$ & \multicolumn{5}{c}{ (DPENDS): $n$ (Ru) Conv. (\%) ee (\%) Configuration } \\
\hline 5 & $0.5: 1$ & 30.6 & 27.1 & $R$ \\
10 & $1: 1$ & 34.1 & 37.0 & $R$ \\
20 & $2: 1$ & 53.8 & 52.3 & $R$ \\
30 & $3: 1$ & 81.5 & 58.3 & $R$ \\
40 & $4: 1$ & 78.2 & 56.1 & $R$ \\
\hline
\end{tabular}

Reaction conditions are same as Table 2 .

表 4 脯氨酸修饰羟基磷灰石做载体时的催化性能

Table 4 Hydrogenation reaction of acetophenone catalyzed by proline modified HAP supported

$\mathrm{RuCl}_{2}(\mathbf{T P P})_{3}$

\begin{tabular}{|c|c|c|c|}
\hline Catalyst & Conv. $(\%)$ & ee $(\%)$ & Configuration \\
\hline D-proline/HAP- $\mathrm{RuCl}_{2}(\mathrm{TPP})_{3}$ & 16.6 & 49.7 & $S$ \\
\hline L-proline/HAP-RuCl ${ }_{2}(\mathrm{TPP})_{3}$ & 28.5 & 53.9 & $R$ \\
\hline $\begin{array}{l}\text { L-proline- } \mathrm{RuCl}_{2}(\mathrm{TPP})_{3} \\
\text { (homogenous) }^{[19]}\end{array}$ & 7.0 & 29.0 & $R$ \\
\hline
\end{tabular}

reaction conditions: $30 \mathrm{mg}$ proline/HAP, $4 \mathrm{mg} \mathrm{RuCl}{ }_{2}(\mathrm{TPP})_{3}, 20 \mathrm{mg}$ $\mathrm{KOH}, n($ acetophenone $)=0.85 \mathrm{mmol} ; V(2$-propanol $)=2.0 \mathrm{~mL}$, $p\left(\mathrm{H}_{2}\right)=5 \mathrm{MPa}, T=313 \mathrm{~K}, t=4 \mathrm{~h}$ 
表 $5(1 R, 2 R)-D P E N D S / H A P$ 负载 $\mathrm{RuCl}_{2}(\mathrm{TPP})_{3}$ 的循环 使用

Table 5 Recycling and reusing the $(1 R, 2 R)$-DPENDS/ HAP supported $\mathrm{RuCl}_{2}(\mathbf{T P P})_{3}$

\begin{tabular}{cccc}
\hline Run & Conv. (\%) & ee (\%) & Configuration \\
\hline 1 & 99.9 & 77.8 & $S$ \\
$2^{\mathrm{a}}$ & 65.5 & 71.1 & $S$ \\
$3^{\mathrm{a}}$ & 15.3 & 49.9 & $S$ \\
$4^{\mathrm{a}}$ & 14.0 & 13.5 & $S$ \\
the filtrate of first run $^{\mathrm{a}}$ & - & - & - \\
\hline Reaction conditions are the same as those in Table 2. ${ }^{\mathrm{a}} 0.43 \mathrm{mmol}$ \\
acetophenone and 2.0 mL 2-propanol were added.
\end{tabular}

明该加氢反应是在手性胺修饰的载体表面进行的.

2.2.6 脯氨酸修饰的羟基磷灰石负载 $\mathrm{RuCl}_{2}(\mathrm{TPP})_{3}$

采用脯氨酸修饰的差基磷灰石负载 $\mathrm{RuCl}_{2}(\mathrm{TPP})_{3}$ 催化苯乙酮进行不对称氢化反应(表 4), 结果发现, 虽然催化剂活性比较低, 但是获得苯乙酮加氢产 物的 ee 值比相应均相催化体系高 ${ }^{[6]}$, 也比文献报道 的脯氨酸修饰的负载 Pd 做催化剂时获得的 ee 值 高 ${ }^{[32-34]}$. 文献报道的脯氨酸修饰的负载 Pd 做催化剂 对苯乙酮的不对称加氢的最高 ee 值在 $28 \%$ 左右, 而 采用本文提供的方法制备的脯氨酸修饰的差基磷灰 石负载 $\mathrm{RuCl}_{2}(\mathrm{TPP})_{3}$ 多相催化剂不对称氢化苯乙酮, 产物的 ee 值可以达到 $50 \%$ 左右, 其结果明显有所提 高. 在脯氨酸修饰的差基磷灰石负载 $\mathrm{RuCl}_{2}(\mathrm{TPP})_{3}$ 催 化体系中, 可能由于载体表面对配合物的结构有所 限制, 使得脯氨酸与金属形成配合物后, 形成的微环 境与均相催化体系不同, 而该微环境的改变可能有 利于产物对映选择性的提高. 结果也进一步说明该 催化体系是多相催化体系, 不对称催化反应是在手 性胺修饰的差基磷灰石的表面进行的.

\subsection{7 催化剂的循环使用}

对催化剂进行了循环实验, 结果如表 5 所示. 反 应完成后催化剂通过离心分离后循环使用, 在不补 加手性修饰剂、膦配体、碱等条件下, 第一次循环可 以获得 $65.5 \%$ 转化率和 $71.1 \%$ 值, 这说明该多相催 化剂通过简单离心分离就能循环使用. 然而在催化 剂的第二次和第三次循环使用中, 产物的 ee 值下降 明显, 其原因可能是在不对称加氢反应中, 形成了 (diamine) $\mathrm{RuH}_{2}(\mathrm{TPP})_{2}$ 活性物种, 其对空气很敏感, 分 离过程中, 催化剂不可避免与空气接触, 这可能是催 化剂在循环过程中失去活性的主要原因. 将反应完 的反应液再加人苯乙酮进行反应, 结果发现底物没 有转化率, 说明没有活性物种流失在溶液中, 对第一 次反应完的反应液进行 ICP 分析, 也没有检测到钌
流失. 由于催化剂用量少, 在目前实验条件下, 尚无 法实现无氧条件下的催化剂与产物分离和循环使 用.

\section{3 结 论}

均相催化剂多相化具备均相催化剂高活性和高 对应选择性的优点, 又具备多相催化剂分离简单, 可 循环使用的优点. 本文根据羟基磷灰石表面具有丰 富差基, 能与带极性基团的有机化合物形成氢键, 通 过共沉淀法制备了手性胺修饰的差基磷灰石. 利用 手性胺修饰的差基磷灰石负载 $\mathrm{RuCl}_{2}(\mathrm{TPP})_{3}$, 成功实 现了采用简单的吸附方法来固载均相催化剂, 达到 了均相催化剂多相化的目的. 利用 $8 \%(1 R, 2 R)$ DPENDS/HAP 负载 $\mathrm{RuCl}_{2}(\mathrm{TPP})_{3}$ 催化苯乙酮的不对 称加氢, 在优化条件下, 可以获得 $99.9 \%$ 的转化率和 $77.8 \%$ 的 ee 值, 其结果略高于三苯基膦和 DPEN 做 配体的均相催化体系在苯乙酮不对称加氢反应中的 结果. 而脯氨酸修饰的差基磷灰石负载 $\mathrm{RuCl}_{2}(\mathrm{TPP})_{3}$ 不对称氢化苯乙酮的 ee 值则明显高于对应的均相 催化体系. 采用各种方法证明了该催化反应体系是 多相催化反应体系, 不对称加氢反应发生在载体表 面. 催化剂循环使用活性降低的可能原因是在不对 称加氢反应中生成的活性物种(diamine) $\mathrm{RuH}_{2}(\mathrm{TPP})_{2}$ 对空气敏感造成的.

\section{References}

1 Noyori, R.; Ohkuma, T. Angew. Chem. Int. Edit., 2001, 40(1): 40

2 Noyori, R. Adv. Synth. Catal., 2003, 345(1-2): 15

3 Vargas, A.; Ferri, D.; Bonalumi, N.; Mallat, T.; Baiker, A. Angew. Chem. Int. Edit., 2007, 46(21): 3905

4 Cheng, H. Y.; Hao, J. M.; Wang, H. J.; Xi, C. Y.; Cheng, X. C.; Cai, S. X.; Zhao, F. Y. J. Mol. Catal. A-Chem., 2007, 278(1-2): 6

5 Jiang, H. Y.; Yang, C. F.; Li, C.; Fu, H. Y.; Chen, H.; Li, R. X.; Li, X. J. Angew. Chem. Int. Edit., 2008, 47(48): 9240

6 Tang, B.; Xiong, W.; Liu, E. R.; Jia, Y.; Wang, J. B.; Chen, H.; Li X. J. Tetrahedron-Asymmetry, 2008, 19(11): 1397

7 Yang, C. F.; Jiang, H. Y.; Feng, J.; Fu, H. Y.; Li, R. X.; Chen, H.; Li, X. J. J. Mol. Catal. A-Chem., 2009, 300(1-2): 98

8 Zhang, D. L.; Yang, C. F.; Feng, J.; Fu, H. Y.; Chen, H.; Li, R. X.; Li, X. J. Acta Phys. -Chim. Sin., 2009, 25(10): 2039 [张定林, 杨朝芬, 冯 建, 付海燕, 陈 华, 李瑞祥, 李贤均. 物理化学学 报, 2009, 25(10): 2039]

9 Yu, H. B.; Hu, Q. S.; Pu, L. Tetrahedron Lett., 2000, 41(11): 1681

10 Takahashi, M.; Haraguchi, N.; Itsuno, S. Tetrahedron-Asymmetry, 2008, 19(1): 60

11 Chiwara, V. I.; Haraguchi, N.; Itsuno, S. J. Org. Chem., 2009, 74 
(3): 1391

12 Chai, L. T.; Wang, W. W.; Wang, Q. R.; Tao, F. G. J. Mol. Catal. A-Chem., 2007, 270(1-2): 83

13 Li, X. G.; Chen, W. P.; Hems, W.; King, F.; Xiao, J. L. Org. Lett., 2003, 5(24): 4559

14 Hu, A. G.; Ngo, H. L.; Lin, W. B. J. Am. Chem. Soc., 2003, 125 (38): 11490

15 Kesanli, B.; Lin, W. B. Chem. Commun., 2004, (20): 2284

16 Hu, A. G.; Yee, G. T.; Lin, W. B. J. Am. Chem. Soc., 2005, 127 (36): 12486

17 Yang, H. Q.; Li, J.; Yang, J.; Liu, Z. M.; Yang, Q. H.; Li, C. Chem. Commun., 2007, (10): 1086

18 Parambadath, S.; Singh, A. P. Catal. Today, 2009, 141(1-2): 161

19 Tanaka, H.; Futaoka, M.; Hino, R. J. Colloid Interface Sci., 2004, 269(2): 358

20 Tanaka, H.; Futaoka, M.; Hino, R.; Kandori, K.; Ishikawa, T. J. Colloid Interface Sci., 2005, 283(2): 609

21 Tanaka, H.; Watanabe, T.; Chikazawa, M.; Kandori, K.; Ishikawa, T. Colloids Surf. A, 1998, 139(3): 341

22 Ei-Hammari, L.; Marroun, H.; Laghzizil, A.; Saoiabi, A.; Roux, C.; Livage, J.; Coradin, T. J. Solid State Chem., 2008, 181(4): 848

23 Borum-Nicholas, L.; Wilson, O. C. Biomaterials, 2003, 24(21): 3671
24 Tanaka, H.; Watanabe, T.; Chikazawa, M.; Kandori, K.; Ishikawa, T. J. Colloid Interface Sci., 1999, 214(1): 31

25 El Shafei, G. M. S.; Moussa, N. A. J. Colloid Interface Sci., 2001, 238(1): 160

26 Matsumoto, T.; Okazaki, M.; Inoue, M.; Hamada, Y.; Taira, M.; Takahashi, J. Biomaterials, 2002, 23(10): 2241

27 Zhang, Y.; Zhao, Y. W.; Xia, C. G. J. Mol. Catal. A-Chem., 2009, 306(1-2): 107

28 Opre, Z.; Ferri, D.; Krumeich, F.; Mallat, T.; Baiker, A. J. Catal., 2006, 241(2): 287

29 Ma, Y. P.; Liu, H.; Chen, L.; Cui, X.; Zhu, J.; Deng, J. G. Org. Lett., 2003, 5(12): 2103

30 Hallman, P. S.; Stephenson, T. A.; Wilkinson, G. Inorg. Synth., 2007, 12: 237

31 Sugiyama, S.; Minami, T.; Hayashi, H.; Tanaka, M.; Shigemoto, N.; Moffat, J. B. J. Chem. Soc. Faraday Trans., 1996, 92(2): 293

32 Tungler, A.; Tarnai, T.; Máthé, T.; Petró, J. J. Mol. Catal., 1991, 67(2): 277

33 Wang, L. L.; Lü, S. J.; Xia, C. G. J. Mol. Catal. (China), 2000, 14 (5): 345 [王来来, 吕世杰, 夏春谷. 分子催化, 2000, 14(5): 345]

34 Chen, X. Y.; Lou, Z. Y.; Qiao, M. H.; Fan, K. N.; Tsang, S. H.; He, H. Y. J. Phys. Chem. C, 2008, 112(5): 1316 